\title{
RADIOCARBON WITH GAS CHROMATOGRAPHY
}

\author{
CHRISTOPHER BRONK RAMSEY and R. E. M. HEDGES
}

Oxford University Radiocarbon Accelerator Unit, 6 Keble Road, Oxford OX1 3QJ, England

\begin{abstract}
In ${ }^{14} \mathrm{C}$ tracer studies, and when looking for modern contamination in archaeological samples, it is often necesquently used for separation of chemical fractions. The accelerator mass spectrometry (AMS) provides the The gas ion source at the Oxford Radiocarbon Accelerator Unit for the first investigations are likely to be ${ }^{14} \mathrm{C}$ the opportunity to measure fractions from a GC instrument directly. Although present results from a pilot study of the peak sensivityes, such a GC-AMS system could find much wider application. We way. We also discuss the
\end{abstract}

\section{INTRODUCTION}

The purpose of this research is to further widen the scope of accelerator mass spectrometry (AMS) in the field of radiocarbon analysis. The ${ }^{14} \mathrm{C}$ isotope is widely used for archaeological dating, environmental tracing at natural abundance and in biological or medical applications at enhanced levels. For simple ${ }^{14} \mathrm{C}$ dating, where there is little contamination, only the proportion of the isotope has to be measured. In all other cases, however, it is the distribution of the isotope that is of greatest interest.

Microprobe ion sources are being developed (see Freeman, Bronk Ramsey and Hedges 1994) to the state where spatial distributions of the ${ }^{14} \mathrm{C}$ isotope can be measured, although not at natural abundance levels. This work is aimed at enabling the chemical distribution to be measured in conjunction with the methods of gas, and possibly liquid, chromatography (see McNair and Bonelli (1968) for a simple introduction to gas chromatography (GC)).

In a GC system, an aliquot of the sample material is injected into a stream of carrier gas. As the gas is swept through a capillary or specially prepared column, the constituents of the gas become separated. A simple detector is then used to record the ejection of the separated aliquots, which appear as peaks on a plot. In an extension of this technique, mass spectrometry (GC-MS) is used to further characterize the constituents of the peaks, often after combustion. Here we show that, by using a gas ion source, of the type found at Oxford, and direct injection, it is possible to measure ${ }^{14} \mathrm{C}$ levels in aliquots of gas of the size that would be produced by an analytical GC.

\section{METHODS}

We use a gas ion source, the details of which have been published elsewhere (Bronk and Hedges 1987, 1989, 1990; Bronk Ramsey and Hedges 1994a). The inlet to this source is a 1-m-long, 0.5$\mathrm{mm}$-diameter capillary through which $\mathrm{CO}_{2}$ is normally flowed. In this application, however, an inert carrier gas is injected into the source through the capillary and the $\mathrm{CO}_{2}$ is mixed with this carrier, simulating the output from a GC system.

The helium carrier gas flow in the various experiments reported here was $c a .0 .01 \mathrm{ml} \mathrm{atm} \mathrm{s}^{-1}$ and was derived from a flow of $1 \mathrm{ml} \mathrm{atm} \mathrm{s}^{-1}$ using a splitter valve. This avoids the problem of handling the very small quantities of $\mathrm{CO}_{2}$ involved directly. The $\mathrm{CO}_{2}$ was introduced into the He stream using the ampule system of the Oxford gas ion source (Bronk Ramsey and Hedges 1994b). 


\section{RESULTS}

The purpose of this work is to evaluate the effect of the inert carrier gas on the performance of the source and to see the effects of resputtering of sample material on the form of the peaks measured and on the cross-contamination between them. This is important, because some samples may contain constituents that differ in ${ }^{14} \mathrm{C}$ concentration by factors of at least 100 . Pulses of $\mathrm{CO}_{2}$ from the new oxalic acid standard (HOxII) and a "dead" $\mathrm{CO}_{2}$ (from anthracite) were injected alternately. Figure 1 shows the measurements from the three isotopes.

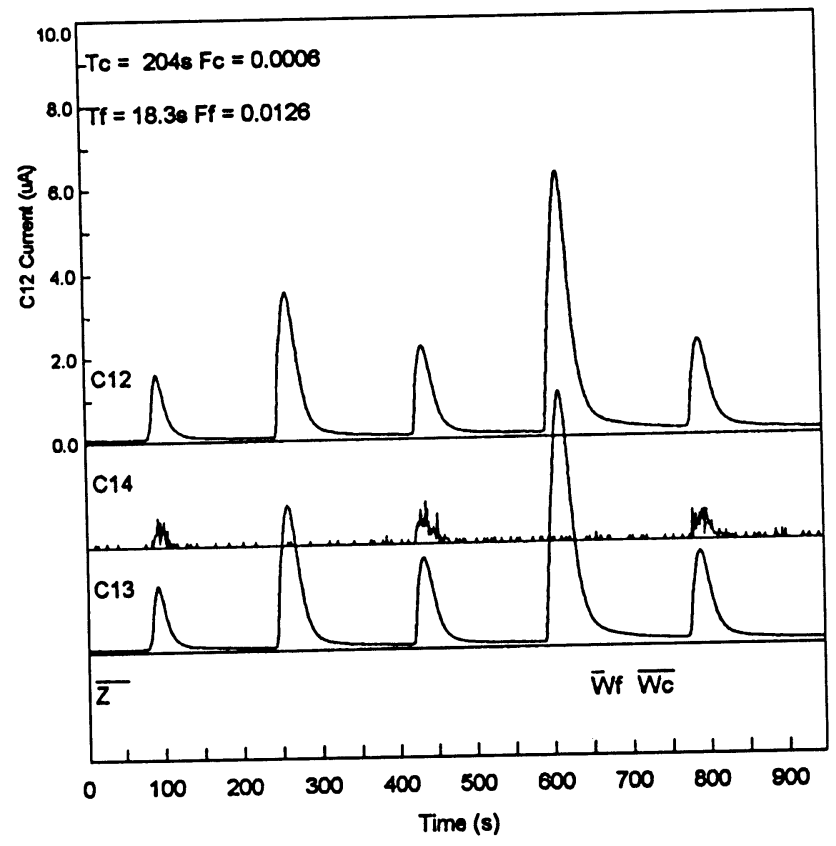

Fig. 1. Trace showing the variation of the three isotopes of carbon with time as pulses of $\mathrm{CO}_{2}$ gas are injected into the source mixed with the $\mathrm{He}$ carrier gas. The pulses alternate between the NBS oxalic acid standard $\mathrm{HOxII}$ and $\mathrm{CO}_{2}$ from anthracite that contains no ${ }^{14} \mathrm{C}$. The target background was measured during the window marked $\mathbf{Z}$, whereas the time constants for the sputter decay $T_{c}$ and gas flow $T_{f}$ were estimated from the current profile in the windows Wc and Wf, respectively.

\section{Peak Sensitivity}

The overall detection efficiency for the gas ion source with the Oxford Accelerator is typically $c a$. $1.5 \%$ (30\% transmission and $5 \%$ overall source efficiency). This normally includes resputtered atoms and, allowing for this, along with some beam attenuation from the carrier gas, the overall efficiency for ions detected a peak window is $c a .1 \%$. It should be stressed, however, that there can be variations in efficiency because of differences in the flow rate of the carrier gas and source conditions.

\section{Sample Sizes}

If the flow rate of the $\mathrm{CO}_{2}$ exceeds a certain value, the changes in the work function of the target reduce the ionization efficiency and the current starts to fall with increasing gas flow. The maximum peak current from the source with short pulses of gas is $c a .8-10 \mu \mathrm{A}$. This corresponds to a maximum sample flow rate of $c a .50 \mathrm{ng} \mathrm{s}^{-1}$ of ${ }^{12} \mathrm{C}$. Typical peak shapes and durations put an upper limit on a measurable aliquot of $c a .1 \mu \mathrm{g}$ of ${ }^{12} \mathrm{C}$. It would be possible to use longer peak durations, but if they are much above $1 \mathrm{~min}$, it is probably more efficient to collect the peak and inject it at constant pressure. Minimum aliquot sizes are more difficult to estimate, but as the background current from a target will be $c a .1 \%$ of the maximum (allowing for some resputtering from previous peaks), a minimum of $10 \mathrm{ng}$ would be reasonable. 
With efficiency as discussed above, the detected masses after acceleration will range from 0.1 to 10 ng ${ }^{12} \mathrm{C}$.

\section{Peak Resolution}

We attribute an appreciable tail on each of the peaks to the embedding and resputtering of material. We can fit this tail to an exponential function of the following form

$$
I_{\text {total }}(t)=I_{\text {gas }}(t)+F_{c} \int_{0}^{t} I_{\text {total }}\left(t^{\prime}\right) e^{-\left(t-t^{\prime}\right) / T_{c}} d t^{\prime}
$$

where $I_{\text {total }}$ is the current measured, and $I_{\text {gas }}$ is the current being generated from the gas as it arrives at the surface of the target. It is then possible to calculate the form of $\mathrm{I}_{\mathrm{gas}}$, as shown in Figure 2 . This level of fitting is clearly ample for discriminating well-separated peaks. However, a small tail remains that might make it difficult to calculate accurate ratios from closely spaced peaks $(<60-s$ peak separation). This smaller tail with a much shorter time constant is probably due to the characteristics of the gas flow into the source. By fitting to a more complicated function

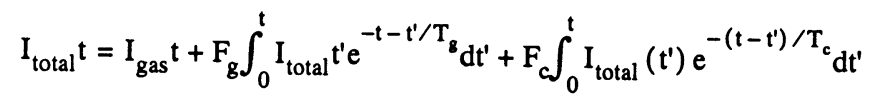

the effect of this smaller tail may be reduced as shown in Figure 3. The disadvantage of such fitting is that it increases the statistical uncertainty on the ${ }^{14} \mathrm{C}$ measurements. There is clearly a direct payoff between statistical accuracy and peak resolution.

\section{Stable Isotope Measurements}

As both the ${ }^{12} \mathrm{C}$ and the ${ }^{13} \mathrm{C}$ currents are measured it is easy to calculate the stable isotope ratios for the peaks. These are shown by the "C1312" line in Figure 3. The oxalic acid $\mathrm{CO}_{2}$ peaks $(0,2$ and 4$)$

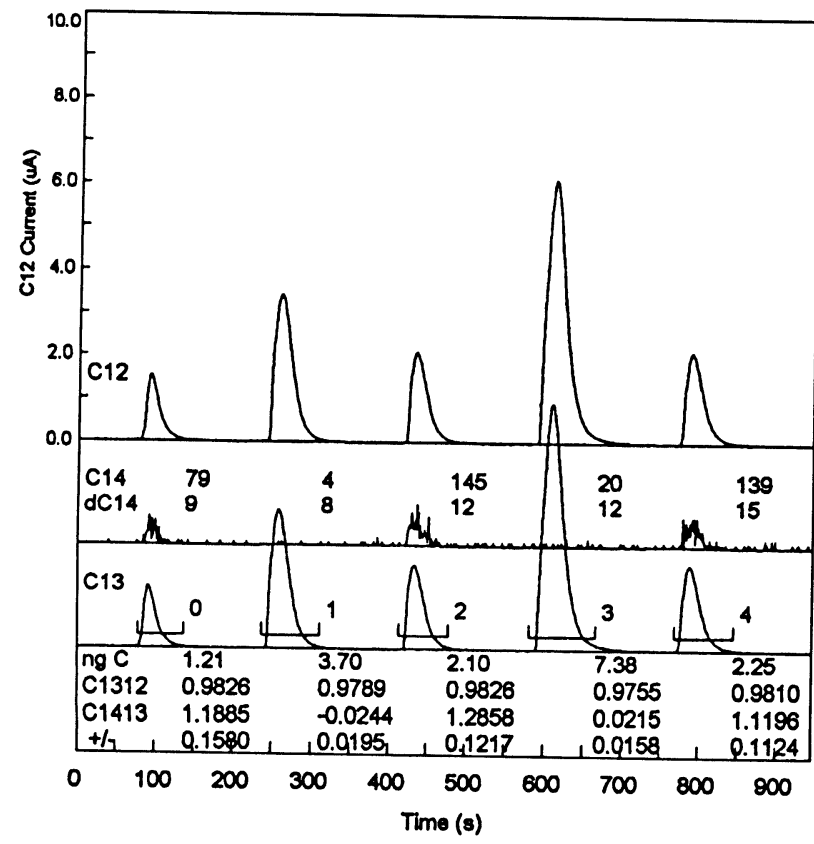

Fig. 2. This trace is derived from that in Fig. 1, taking into account the background current and the sputter decay but not the gas flow. The numbers given in the $\mathrm{C} 14$ row are the absolute number of ${ }^{14} \mathrm{C}$ atoms detected within each window and the $\mathrm{dC} 14$ gives the subtraction that has been made for the background and sputter decay. The ng $\mathrm{C}$ row indicates the mass of ${ }^{12} \mathrm{C}$ detected, after the accelerator, and the $\mathrm{C} 1312$ figures give the ${ }^{13} \mathrm{C} /{ }^{12} \mathrm{C}$ ratio $\times 100$. The $\mathrm{C} 1413$ and $+/$ - rows give the ${ }^{14} \mathrm{C} /{ }^{13} \mathrm{C}$ ratio and associated uncertainty (both $\times 10^{-12}$ ). 


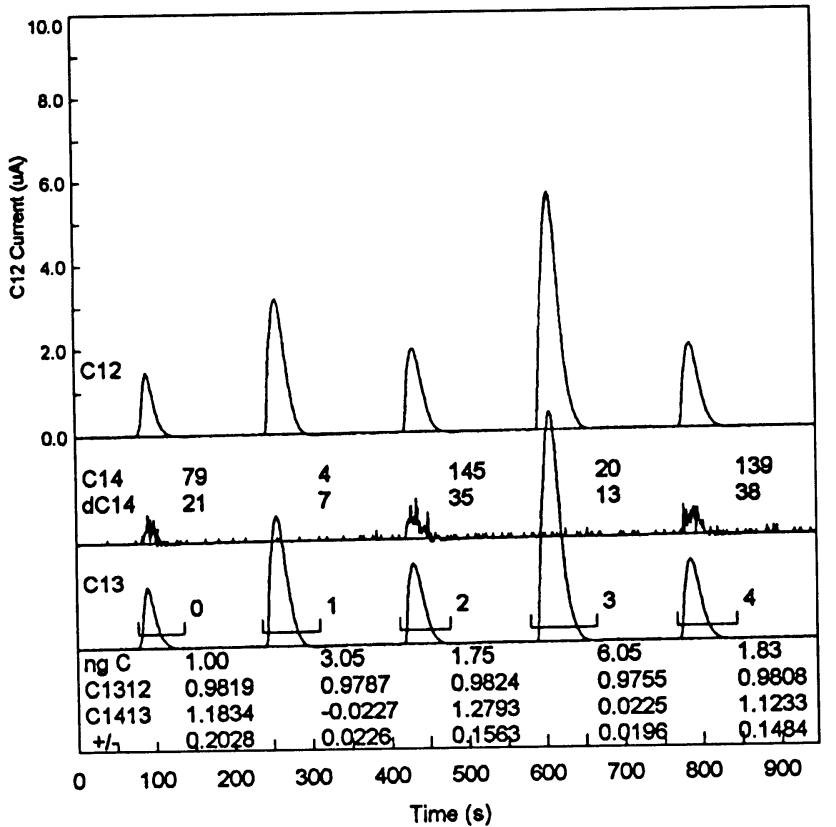

Fig. 3. Similar to Fig. 2, but including the corrections for the flow of gas into the source. The peak shapes are easier to resolve, but the uncertainties in the ${ }^{14} \mathrm{C} /{ }^{13} \mathrm{C}$ ratio have increased substantially.

all have ratios lying within $2 \%$, with an average of 0.9817 . The "dead" $\mathrm{CO}_{2}$ peaks have an average of 0.9771 , indicating a difference of $-4.6 \%$, which compares well with $-5.28 \%$ from measurements on a stable isotope mass spectrometer. These results indicate that the accuracy of isotope ratios should be within $2 \%$. If the peak height is allowed to approach the maximum, this accuracy is somewhat compromised due to space-charge effects on the ${ }^{12} \mathrm{C}$ beam.

\section{${ }^{14} \mathrm{C}$ Measurements}

Figure 3 shows the results of the ${ }^{14} \mathrm{C}$ measurements on the samples of "dead" $\mathrm{CO}_{2}$ and those from the oxalic acid standard. The ${ }^{14} \mathrm{C}$ ratios are at the expected levels (the oxalic acid standard normally gives ratios of $c a .1 .16$ with this source and accelerator). The uncertainty associated with the ratio measurements depends on the amount of deconvolution performed. For example, for peak 4 , which contains $c a .2 .6 \mathrm{ng}$ of ${ }^{12} \mathrm{C}$, as measured after the accelerator, the precision is $c a .10 \%$ once the target background correction has been made, which is almost purely statistical in nature. Making the corrections for the sputter tail, which is necessary to check for the effect of cross-contamination, increases this error by a factor of $c a$. 1.1. If the correction is also made for the gas flow into the source, this is further multiplied by 1.2 .

The largest and fundamental problem is the statistical accuracy of the measurements. Figure 4 shows the limiting measurement accuracy for various quantities of ${ }^{12} \mathrm{C}$ at different levels of enrichment. These quantities would need to be injected into the source $c a .100$ times. Another approach to this problem is to consider the amount of ${ }^{14} \mathrm{C}$ needed to register a peak. The minimum number of ${ }^{14} \mathrm{C}$ atoms that will register significantly above the background is $c a$. 10; thus $c a .1000$ would have to be injected into the source. If the ratio is to be measured to a precision of $10 \%, c a .100$ must be measured and 10,000 injected; for $1 \%$ ca. 10,000 measured and 1,000,000 injected. 


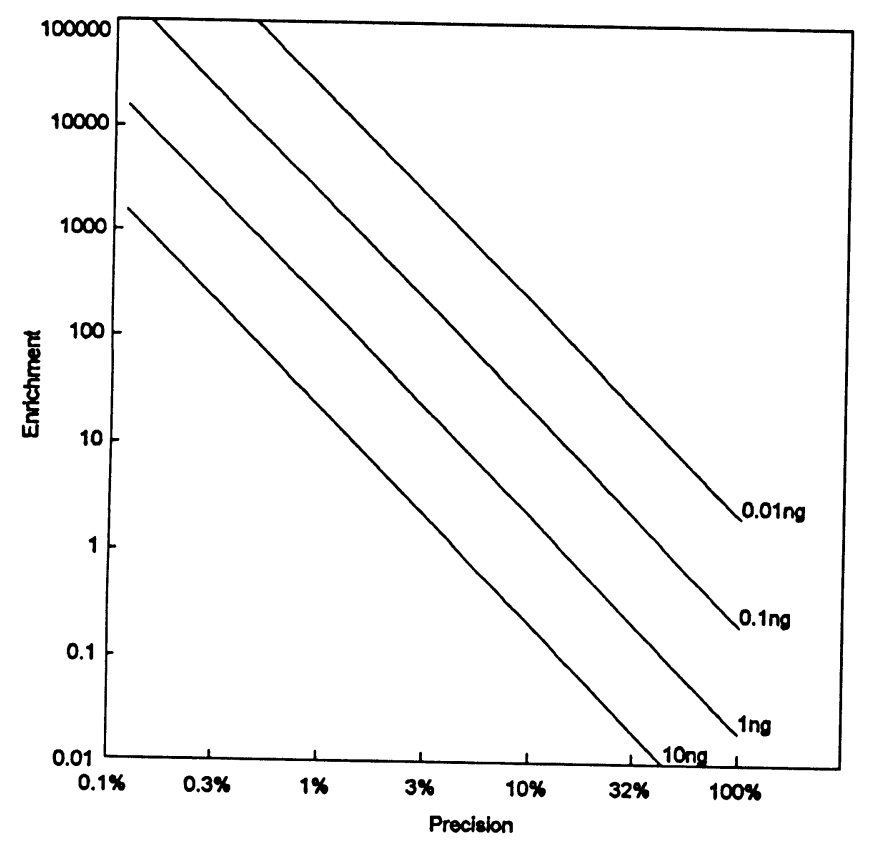

Fig. 4. Theoretical measurement precision limit for samples of different sizes and enrichments. The enrichment of 1 corresponds to modern levels of ${ }^{14} \mathrm{C} .10 \mathrm{ng}$ of measured ${ }^{12} \mathrm{C}$ is probably the largest aliquot size for this method. Because the overall measurement efficiency is $c a .1 \%$, ca. 100 times as much material as this would have to be injected into the source to achieve these precisions.

\section{Cross-Contamination}

The uncertainties quoted for the ratios on the dead $\mathrm{CO}_{2}$ (peaks 1 and 3 ) also include an element from the cross-contamination corrections and are $c a .2 \%$ of the oxalic acid standard ratio. For samples that contain some modern material, ratios should be measurable down to $c a .5 \%$ of the modern ratio (for peaks of comparable size and close separation). A practical limit would be that no peak should contain $>50$ times the number of ${ }^{14} \mathrm{C}$ atoms than any other for which a measurement is required.

\section{FUTURE DEVELOPMENTS}

To make a GC-AMS system suitable for general use, it should be connected directly to the source. The main complication with this is the need for the output of the GC to be oxidized before passing to the source. Depending on the nature of the material to be analyzed, it may be desirable to trap out the water vapor produced by such a combustion. We do not foresee any particular technical difficulties with this because it is the method already used routinely with isotope ratio mass spectrometry (GC-IRMS). Looking farther ahead, it should also be possible to link the system to a high-pressure liquid chromatography (HPLC) system, if the carrier solvent can be sufficiently separated from the sample before combustion and injection.

\section{Potential APPLiCations}

Three main types of measurement are susceptible to this technique: 1$)^{14} \mathrm{C}$ dating of very small samples; 2) environmental studies of atmospheric ${ }^{14} \mathrm{C}$; and 3$)^{14} \mathrm{C}$ tracers for biological or biomedical research.

\section{Radiocarbon Dating of Very Small Samples}

Where the sample levels are very low $\left(<1 \mu \mathrm{g}{ }^{12} \mathrm{C}\right)$, sample preparation and handling become extremely difficult. Removal of contaminants by GC analysis is a well-established technique and is 
ideal for small samples. This method allows the ${ }^{14} \mathrm{C}$ measurement to be carried out directly with purification, thereby allowing the age of any contaminant or other fractions to be determined at the same time. The main limitation is statistical accuracy, which is unlikely to be $>10 \%$. However, for applications in which this is not an overriding concern, the derived dates would still be of value.

Related to this is the investigation of contaminants themselves. $\mathrm{GC}$ analysis allied to ${ }^{14} \mathrm{C}$ dating would allow contaminants of a radically different age from the actual sample to be identified. Preparative $\mathrm{GC}$ analysis designed to collect enough of the sample for accurate ${ }^{14} \mathrm{C}$ analysis could then be performed using this information.

\section{Environmental Studies}

Another area in which the levels of ${ }^{14} \mathrm{C}$ need to be measured is in environmental monitoring of trace constituents of the atmosphere. Products of fossil fuel and biogenic components can be directly discriminated by the ${ }^{14} \mathrm{C}$ content. Leakage of ${ }^{14} \mathrm{C}$ produced by neutron bombardment of nitrogen in the nuclear industry can also be a useful tracer for possible leakage and for those parts of the biosphere directly affected. In these cases, ${ }^{14} \mathrm{C}$ measurement accuracy is not critical but the ability to work with very small quantities is essential.

\section{Radiocarbon Tracers}

The third area of importance is in ${ }^{14} \mathrm{C}$ tracer studies. In principle, these enable very small quantities of a substance to be detected (down to 1000 atoms for $100 \%{ }^{14} \mathrm{C}$ ). The ability to combine this with $\mathrm{GC}$ analysis would make a tool for uniquely sensitive chemical analysis.

\section{CONCLUSION}

The Oxford gas ion source has been used to demonstrate the feasibility of using such a system for GC-AMS measurements. Given the currents available and the sample sizes envisaged, we cannot measure ${ }^{14} \mathrm{C}$ dates accurately using this method. The best precisions available are likely to be $c a$. $10 \%$ for modern material. For the largest peaks, background levels are likely to be of the order of 1$2 \%$ if there is modern material in some of the peaks. Enriched ${ }^{14} \mathrm{C}$ can be measured to a much higher precision and, from the stable isotope ratios, the accuracy of such measurements should be within $0.2 \%$. This level of performance should be useful for approximate dating and distinguishing whether individual peaks have radically different ${ }^{14} \mathrm{C}$ contents. The most likely areas of application of this technique are biomedical and environmental studies with ${ }^{14} \mathrm{C}$ tracers, where as few as 1000 atoms could be detected, or for discrimination between biogenic and fossil-fuel sources.

\section{REFERENCES}

Bronk, C. R. and Hedges, R. E. M. 1987 A gas ion source for ${ }^{14} \mathrm{C}$ dating. Nuclear Instruments and Methods in Physics Research B29: 45-49.

1989 Use of the $\mathrm{CO}_{2}$ source in radiocarbon dating by AMS. In Long A., Kra, R. S. and Srdox, D., eds., Proceedings of the 13 th International ${ }^{14} \mathrm{C}$ Conference. Radiocarbon 31(3): 298-304.

Bronk, C. R. and Hedges, R. E. M. 1990 A gaseous ion source for routine AMS ${ }^{14} \mathrm{C}$ dating. Nuclear Instruments and Methods in Physics Research B52: 322-326.

Bronk Ramsey, C. and Hedges, R. E. M. 1994a Carbon dioxide sputter source development at Oxford. $\mathrm{Nu}$ clear Instruments and Methods in Physics Research B92: 100-104.

1994b Gas handling systems for ${ }^{14} \mathrm{C}$ dating by AMS. Nuclear Instruments and Methods in Physics Research B92: 105-110.

Freeman, S. P. T., Bronk Ramsey, C. and Hedges, R. E. M. 1994 Imaging AMS. Nuclear Instruments and Methods in Physics Research B92: 231-236.

McNair, H. M. and Bonelli, E. J. 1968 Basic Gas Chromatography. Berkeley, California, Varian Instruments. 